\title{
AUTORREGULAR EL DESEMPEÑO ESCRITO EN INGLÉS AL RECOMENDAR TEXTOS EN LA MEDIATECA DE LENGUAS DEL BACHILLERATO
}

\author{
SELF-REGULATING WRITING PERFORMANCE IN ENGLISH \\ WHEN RECOMMENDING TEXTS AT A HIGH SCHOOL \\ LANGUAGE MEDIA RESOURCE CENTER
}

\author{
Ernesto Hernández Rodríguez \\ Universidad Nacional Autónoma de México, \\ Escuela Nacional Preparatoria, Plantel 3 Justo Sierra
}

RESUMEN

Este trabajo presenta una propuesta didáctica enmarcada en las asesorías que se ofrecen en una mediateca de lenguas a nivel bachillerato con el fin de fomentar la autorregulación de la escritura. En la propuesta los alumnos redactan, revisan y editan la recomendación de un texto narrativo en inglés como lengua extranjera con el acompañamiento de un asesor. La metodología incluye la toma de acuerdos entre los alumnos y el asesor sobre las características de las recomendaciones escritas. También comprende la revisión y edición de los textos apoyadas por el corrector ortográfico y gramatical de Word, así como por las observaciones del asesor y, cuando es posible, de otro docente. El propósito de esta propuesta es propiciar en los alumnos prácticas relacionadas con los estándares de textualidad, el tipo de texto empleado en una recomendación y la representación textual del autor y los interlocutores. Con estas experiencias de textualidad los alumnos pueden reflexionar sobre sus desempeños escritos y autorregularlos para tratar de lograr una última versión mejorada de las recomendaciones.

El desempeño textual de los estudiantes se analiza a lo largo del artículo a partir de las versiones iniciales, intermedias y finales de sus recomendaciones, las cuales presentan evidencias de autorregulación en la escritura, vinculada con la retroalimentación recibida. Asimismo, se describen las actitudes, decisiones y estrategias tomadas por los estudiantes para tratar de resolver las dificultades de escritura enfrentadas. Un hallazgo de la investigación es el predominio del interés de los estudiantes por la gramática y la ortografía en las diferentes etapas de revisión de sus textos. 
PALABRAS CLAVE: intencionalidad; autorregulación; escritura; inglés como lengua extranjera; nivel medio superior; retroalimentación

\section{Abstract}

This work presents a didactic proposal as part of the advisory services provided by a high-school language media center aimed at encouraging students to self-regulate their performance when writing in English as a foreign language. The proposal prompts the students to write, revise and edit a recommendation in English of a narrative text, with support from an advisor. The methodology includes that the students and the advisor agree on the characteristics of the written recommendation and then revising and editing the texts based on feedback provided by the spelling and grammar checker in the Word software, the advisor and, whenever possible, another teacher. The aim is to make the students adopt good writing practices related to textuality standards, the text type appropriate for recommendations and the representation of authors and interlocutors in the text. Based on these textuality experiences, students can ponder on and self-regulate their writing performance to attain an improved final draft.

The article examines the students' writing performance based on the earliest, intermediate and final drafts of their recommendations; these showed evidence of self-regulated writing linked to the feedback received. The attitudes, decisions and strategies adopted by students to overcome the difficulties faced during writing are also described. A noticeable finding is that the students' major concerns, over the various revision stages of their texts, were grammar and spelling.

KEYWORDS: intentionality; self-regulation; writing; English as a foreign language; high school level; feedback 


\section{INTRODUCCIÓN}

Los estudiantes de bachillerato se enfrentan a múltiples problemáticas relacionadas con sus desempeños escritos, en lenguas materna y extranjera, en diversas actividades de sus asignaturas. En general, los estudiantes requieren de un trabajo constante para el desarrollo de la redacción en actividades retroalimentadas. En estas prácticas es fundamental la revisión y la edición de los escritos en interacciones que fomenten la reflexión y la autorregulación del desempeño. Una manera de promover este tipo de prácticas es invitar a los estudiantes a recomendar lecturas para compartir sus puntos de vista con otros por escrito.

En la enseñanza del inglés como lengua extranjera, la lectura de textos y su recomendación por escrito implican tareas relacionadas con esta lengua en la comprensión de la lectura, el manejo de los recursos de redacción, el texto requerido para una recomendación, la gramática, el vocabulario y la representación del autor y los lectores para expresar una opinión y recomendar el texto.

En este sentido, este artículo presenta una propuesta de prácticas de autorregulación reflexiva del desempeño escrito en inglés para recomendar lecturas, seleccionadas por los estudiantes, en las asesorías de una mediateca de lenguas del bachillerato. Estas actividades tienen como finalidad propiciar la redacción, la revisión y la edición de los escritos con la idea de retomarlos para tratar de mejorarlos en las siguientes versiones redactadas. La retroalimentación incluye el apoyo mediante el corrector ortográfico y gramatical del procesador de textos Word, las observaciones y sugerencias del asesor y, de ser posible, de otro docente. Esta propuesta se puso en práctica en la Escuela Nacional Preparatoria, plantel 3, Justo Sierra, ubicada en la Ciudad de México, con 6 alumnos en febrero de 2015. En este trabajo se presentan los resultados de esta experiencia.

En el apartado siguiente a esta introducción, §2, se presentan los enfoques en los que se fundamentó la propuesta y la caracterización de la escritura de los estudiantes que participaron en las asesorías. Estos incluyen los enfoques de estándares de textualidad (De Beugrande \& Dressler, 1997), la regulación de la intencionalidad y el desempeño lingüístico (Bausela Herreras, 2007; Beuchot, 2015), las estrategias compensatorias para enfrentar y tratar de resolver dificultades (Oxford, 1990; Martín Leralta, 2006; Palapanidi, 2016) y la representación textual de autores y lectores para recomendar una lectura (Hyland, 2005). 
En §3, se inicia la descripción de la puesta en práctica de la propuesta con la caracterización del nivel de dominio de los estudiantes que participaron, la cual está centrada en sus habilidades de lectura y, principalmente, de escritura. Además, se considera el contexto escolar del inglés como lengua extranjera en la institución.

En §4, se presenta el diseño y la metodología de la propuesta de asesoría, incluyendo, en primer lugar la sesión inicial, que tiene como fin sensibilizar y tratar de motivar a los estudiantes a recomendar lecturas y establecer acuerdos sobre las características de la recomendación escrita de un texto, y, en segundo lugar, la dinámica de asesorías orientadas a escribir y retomar los textos para revisarlos y tratar de mejorarlos en las siguientes versiones.

El apartado $\$ 5$ se dedica a la descripción de los textos de los alumnos centrándose en la caracterización de la intencionalidad y del desempeño conforme a los criterios conceptuales expuestos en $\S 2$. Los ejemplos de las versiones iniciales, intermedias y finales incluyen los puntos esenciales de la retroalimentación con el corrector ortográfico y gramatical del procesador de textos Word, con el asesor y, cuando fue posible, con otro docente. Asimismo, esta sección presenta las decisiones de textualización de los estudiantes, con el énfasis que pusieron en determinados estándares de textualidad, las estrategias empleadas para tratar de mejorar la escritura y las reflexiones escritas de quienes desearon opinar sobre su desempeño. Finalmente, el artículo cierra con las conclusiones en $§ 6$.

\section{ENFOQUE}

Consideramos que las prácticas interactivas, reflexivas y autorreguladas de escritura, revisión y edición de textos para recomendar lecturas tienen lugar cuando los autores y los lectores toman en cuenta las características y expectativas de recepción de un texto en determinado entorno y situación comunicativa.

La propuesta de prácticas de redacción, revisión y edición de textos que se presentan en este artículo tiene su fundamento, en primer lugar, en los estándares de textualidad planteados por De Beugrande y Dressler (1997): cohesión, coherencia, intencionalidad, informatividad, situacionalidad, aceptabilidad e intertextualidad. La cohesión involucra la construcción formal, gramatical y la estructuración del texto. La coherencia permite que los mensajes expresados sean claros y entendibles. La intencionalidad corresponde a la actitud de quien 
escribe para tratar de lograr los propósitos que desea expresar. La informatividad está relacionada con los contenidos abordados. El estándar de situacionalidad nos permite ubicar la comunicación en un entorno o contexto. La aceptabilidad es el criterio para establecer si un escrito es pertinente y cumple con los criterios de propiedad en un género y situación comunicativa y está relacionada con la actitud del receptor en determinado ambiente. La intertextualidad es el establecimiento de relaciones de un escrito con otros textos o lecturas realizadas, por ello está estrechamente vinculada con el estándar de informatividad. La propuesta de prácticas de redacción, revisión y edición de recomendaciones que aquí se presenta pretende propiciar en el alumno, en la medida de lo posible, la toma de conciencia acerca de estos criterios al revisar y valorar su desempeño con el fin de realizar las modificaciones en cada versión redactada.

La propuesta se basa, asimismo, en el reconocimiento de la reflexividad textual (Hyland, 2005) de quien escribe cuando toma en cuenta el contenido de su texto, el contexto y los lectores. Siguiendo a Beuchot (2015), en la revisión y edición textual tomamos en cuenta la interpretación y la intencionalidad en aquello que los estudiantes tratan de expresar, pero que muchas veces no logran plasmar por escrito. Consideramos además que la revisión y edición reflexivas favorecen la toma de decisiones al modificar el texto para mejorarlo. En este sentido, Torre Puente considera que en la reflexión sobre el desempeño, "enseñar a preguntarse, a auto vigilarse, a analizar el fallo en los hábitos comportamentales, a detenerse y a analizar qué es lo que se está haciendo, es el punto de partida para la consecución de la autorregulación" (1992: 55). Otros autores que siguen esta línea son Wenden (1986), Dickinson (1987) y Bausela Herreras (2007), quienes estudian la toma de conciencia de las habilidades, estilos de aprender y autorregulación del desempeño lingüístico. ${ }^{1}$ Oxford (1990), Martín Leralta (2006) y Palapanidi (2016) van más allá de la reflexión sobre el desempeño al proponer el manejo de estrategias compensatorias del desempeño en la búsqueda de opciones para tomar decisiones alternas encaminadas a resolver dificultades en la expresión. Oxford (1990) destaca que mediante estas estrategias tenemos la posibilidad de resolver fallas en el desempeño o carencias de conocimiento para lograr una comunicación auténtica:

1 En Castelló, Bañales Faz y Vega López (2010) podemos revisar un panorama de los enfoques de la autorregulación de la escritura. 
Compensation strategies aid learners in overcoming knowledge gaps and continuing to communicate authentically; thus, these strategies help communicative competence to blossom. As learners' competence grows, strategies can act in specific ways to foster particular aspects of that competence: grammatical, sociolinguistic, discourse, and strategic elements (Oxford, 1990: 9). ${ }^{2}$

En esta perspectiva, consideramos que la autorregulación en la revisión y edición textual favorece la toma de decisiones reflexivas para modificar los recursos de textualización y tratar de mejorar la escritura en determinado contexto. En las decisiones autorreguladas, el estudiante somete a juicio y prueba sus propios recursos de escritura para determinar si el texto responde a la situación comunicativa y para hacer las modificaciones pertinentes con la idea de mejorarlo. La textualización corresponde al manejo de conocimientos y recursos formales, comunicativos, contextuales y culturales involucrados en la práctica de plasmar el pensamiento por escrito. Los estudiantes requieren de orientación para aprender a reflexionar sobre su desempeño, tomando en cuenta los criterios de escritura relacionados con los estándares de textualidad, el tipo de texto y la representación del autor y los interlocutores.

El propósito de las asesorías es fomentar las estrategias de autorregulación reflexiva sobre el desempeño en la revisión y edición de los escritos. La interacción y la retroalimentación favorecen la escritura significativa de las recomendaciones que, una vez terminadas, son difundidas entre la comunidad de la mediateca. Ivanič (1998) propone que los alumnos toman decisiones de escritura que manifiestan la conformación de identidad y la socialización de sus escritos, al asumir determinada actitud y postura ante sus interlocutores. Tomando esto en cuenta, consideramos que se busca que la retroalimentación por parte de los asesores promueva una actitud positiva, crítica y reflexiva de la revisión y edición textuales.

2 "Las estrategias compensatorias ayudan a los aprendientes a superar vacíos de conocimiento y a continuar comunicándose de manera auténtica; por lo tanto, estas estrategias ayudan a que la competencia comunicativa florezca. En tanto que la competencia de los aprendientes crece, las estrategias pueden actuar de maneras específicas para fortalecer aspectos particulares de determinada competencia: gramatical, sociolingüística, discursiva y de elementos estratégicos". [ $L a$ traducción es del autor. N. del E.] 


\section{CARACTERIZACión DEL NIVEL DE DOMinio DE LA LENGUA DE LOS} ESTUDIANTES

Los talleres de la mediateca ofrecen opciones extracurriculares para el desarrollo de habilidades comunicativas en un entorno de aprendizaje autorregulado. En general, resulta difícil y polémico establecer un nivel de dominio de la lengua de los estudiantes que asisten a la mediateca debido a las distintas trayectorias escolares de los alumnos de la Escuela Nacional Preparatoria. Algunos estudiantes leen en inglés, pero no se expresan oralmente; otros comprenden fragmentos de conversaciones, sin embargo, no se comunican de manera oral o escrita.

Por otra parte, los materiales más recientes de la mediateca indican los niveles de la lengua extranjera conforme a los criterios acordados por el Marco Común Europeo de Referencia de las Lenguas (MCER): A1, A2, B1, B2, C1 y C2. Esta propuesta contempla determinados indicadores de desempeño para el manejo, predominantemente integral, de las habilidades de lectura, escritura, expresión oral y comprensión auditiva. El dominio de solo una habilidad corresponde a un nivel parcial, por ejemplo, de A1 o A2 (Council of Europe for Cultural Cooperation Education Committee, 2001). En el acervo de la mediateca también existen textos con el anterior criterio de niveles: introductorio, básico, intermedio y avanzado, así como métodos para el desarrollo de determinadas habilidades con propósitos específicos. Esta coexistencia de criterios sobre los niveles del manejo de la lengua da lugar a confusiones en los intentos por caracterizar el desempeño lingüístico de los estudiantes y los materiales del acervo de la mediateca. Esta situación es motivo de una investigación independiente sobre el tema. Por ello, en este trabajo únicamente se considera el criterio de desempeño inicial o básico en las habilidades de lectura y, principalmente, de escritura.

La puesta en práctica de esta propuesta de experiencias de escritura se llevó a cabo en febrero de 2015 en el taller extracurricular de recomendación escrita en inglés de lecturas. Al taller asistieron seis alumnos de 15 años de la Escuela Nacional Preparatoria, plantel 3. Estos alumnos cursaron la asignatura de inglés en la escuela secundaria y en la preparatoria la cursaban como lengua extranjera en el primer curso. En el nivel inicial se espera que los alumnos manejen el presente y el pasado simples, así como la lectura de textos básicos (Escuela Nacional Preparatoria, 1996). 
No se realizó una caracterización ni evaluación del nivel de dominio de habilidades lingüísticas de los estudiantes que asistieron al taller, ya que el propósito era que participaran en la experiencia de escritura distintos tipos de alumnos, independientemente de su trayectoria escolar y lingüística. Sin embargo, fue posible observar que los alumnos que participaron en el taller presentaban un dominio de la lengua extranjera predominantemente inicial, centrado en la comprensión lectora y en ejercicios gramaticales, sin desarrollo de habilidades comunicativas conversacionales ni de redacción de textos. Estos alumnos se enfrentaron por primera vez a la experiencia de recomendar una lectura en lengua extranjera en un ambiente escolar, en este caso, a los distintos lectores que asisten a la mediateca.

Debido a la dificultad para establecer determinado nivel de dominio de la lengua y a que el centro de interés estaba puesto en la expresión escrita, la lengua de instrucción oral en el taller fue el español para la interacción, las asesorías y la reflexión sobre la escritura y el desempeño. En este taller el inglés oral se introduce gradualmente en la asesoría individual únicamente cuando determinado estudiante presenta un desarrollo considerable de esta habilidad. Sin embargo, la comunicación oral con los seis estudiantes en esta ocasión fue en español.

\section{Diseño Y METOdOlogía DE LA PROPUESTA DE ASESORÍA}

En una sesión inicial, los alumnos discutieron y reflexionaron sobre la escritura de recomendaciones de lecturas en inglés, con el propósito de que reconocieran la utilidad de esta práctica y que asistieran a las asesorías para realizar este tipo de escritos. Con la finalidad de propiciar la interacción, se les plantearon las siguientes preguntas:

1) ¿Cuál puede ser la utilidad de recomendar lecturas a quienes asisten a la mediateca?

2) ¿Cuál puede ser la utilidad de escribir recomendaciones en inglés de las lecturas de la mediateca?

Posteriormente, los alumnos leyeron y comentaron oralmente un texto que se les proyectó en una pantalla: "Nasreddin and the professor" (Hill, 2007: 8-9) (véase Anexo 1). Durante la actividad, se les apoyó en la comprensión del vocabula- 
rio y el contenido. Después comentaron acerca de la manera en que podían escribir la recomendación a partir de las siguientes preguntas:

3) ¿Cómo recomendarías por escrito este texto a quienes asisten a la mediateca?

4) ¿Qué tomarías en cuenta para escribir tu recomendación?

Como resultado, los aspectos más mencionados fueron: opinar, expresar el gusto, el interés y no contar el final de la historia. A continuación, se propuso a los alumnos lograr acuerdos sobre las características de estos escritos. Después se les sugirió considerar los siguientes aspectos: tomar en cuenta al lector, dirigirse a este para convencerlo y escribir en primera persona del singular (yo). Además, se les mostró dos ejemplos de recomendaciones escritas por estudiantes en la mediateca en el ciclo escolar anterior (véase Anexo 2). La revisión de estos textos, como referentes y experiencias previas, sirvió para que los estudiantes tuvieran una idea sobre cómo valorar distintos desempeños, sobre cómo debe escribirse una recomendación de lectura y para reflexionar posteriormente en torno a su propio desempeño. Después, se tomaron los siguientes acuerdos para la redacción de las recomendaciones:

- Presentar información adecuada: la necesaria, no contar de más, no contar el final.

- Mencionar aspectos generales de la lectura: autor, título.

- Tener una opinión clara, expresar puntos de vista.

- Tomar en cuenta al lector: dirigirse al lector para atraerlo.

- Presentar ejemplos (probaditas de la lectura) para tratar de animar al lector.

- Ser breve y crear un texto interesante.

- Escribir un texto gramatical y ortográficamente cuidado.

En las asesorías individuales, cada estudiante decidió, con la guía del asesor, los recursos, los tiempos de lectura y escritura y sus propias estrategias para redactar las recomendaciones. Cinco estudiantes seleccionaron textos de nivel básico y uno prefirió de nivel intermedio (en el Anexo 1 se presentan los textos escogidos por los estudiantes). Una vez que los alumnos escribieron la primera versión de la recomendación, la transcribieron mediante el procesador de textos Word. Posteriormente, 
se empleó la función de revisión ortográfica y gramatical de Word, que muestra los errores ortográficos subrayados en rojo y los gramaticales, en verde. Sin embargo, el programa no indica errores de selección léxica, ya que para identificarlos se requiere conocer la contextualización del contenido y la intención; la función aplicada se limita solo a la gramática y la ortografía. Luego, los alumnos modificaron lo que consideraron pertinente. Cabe señalar que, en general, los estudiantes no están acostumbrados a revisar ni a seleccionar de manera crítica las opciones sugeridas por esta aplicación del procesador de textos. Por ello, se les orientó sobre su funcionamiento para que pudieran aprovecharla al intentar escribir una versión mejorada. Al final se les pidió que, en español, escribieran sus opiniones sobre esta actividad y sobre sus desempeños.

En otra fase de asesoría, los estudiantes recibieron comentarios y sugerencias del profesor acerca de sus escritos y, cuando fue posible, también los de otro asesor. Se les motivó a reflexionar sobre estas observaciones y su utilidad, así como sobre el apoyo de la función de revisión y corrección del programa Word. Finalmente, a cada estudiante se le hicieron sugerencias para organizar un plan de trabajo en asesoría. El propósito era que desarrollaran miradas críticas de autorregulación y valoración del desempeño.

Con el fin de ayudar a los alumnos a mejorar sus escritos, se diseñó y observó una metodología de procedimiento de las asesorías basada en las siguientes consideraciones: a) el asesor recuerda al estudiante que tome en cuenta los acuerdos para la redacción de los escritos, b) el estudiante revisa el desempeño en su primera versión y recibe observaciones y sugerencias relacionadas con los estándares de textualidad y con cada uno de los acuerdos, c) el asesor da retroalimentación al estudiante sobre los cambios realizados y propicia la reflexión sobre el desempeño al destacar los logros y hacer sugerencias sobre los aspectos que requieren mejoría, d) cuando el estudiante no logra resolver alguna dificultad, el asesor sugiere recursos alternativos que ayuden a la autorregulación del desempeño, por ejemplo, emplear otras palabras o estructuras más sencillas, e) el asesor propone la consulta de materiales impresos y en línea del acervo y recomienda la revisión con el corrector ortográfico y gramatical, f) una vez que el estudiante intentó mejorar y reflexionó sobre su desempeño, recibe explicaciones concretas para determinados aspectos no resueltos, g) el alumno recibe, cuando es posible, asesoría de otro docente o asesor, h) el alumno escribe las versiones que considera necesarias y decide cuál es la última versión mejorada, 
posteriormente el asesor hará observaciones finales e i) el asesor apoya al estudiante para que aprenda a recibir retroalimentación encaminada a la reflexión y autorregulación del desempeño.

\section{SEgUimiEnTo DEL DESEMPEÑo EN LA REVISIÓN Y EDICIÓN DE LOS TEXTOS}

La revisión y la edición de los textos a partir de la retroalimentación obtenida con el procesador ortográfico y gramatical de Word y la del asesor propician prácticas reflexivas de regulación de la escritura de los estudiantes. Durante las asesorías se retoman en todo momento los acuerdos sobre las características de la recomendación escrita de un texto, con el propósito de propiciar la reflexión y autorregulación del desempeño en la revisión y edición de los escritos. Asimismo, las orientaciones de redacción están centradas en criterios de escritura relacionados con los estándares clásicos de textualidad de De Beugrande y Dressler (1997), el tipo de texto requerido y la necesidad de representación textual del autor y los interlocutores. En este trabajo se presentan únicamente las versiones completas iniciales, intermedias y finales de tres estudiantes (textos: $\mathrm{t} 1 \mathrm{~m}, \mathrm{t} 2 \mathrm{~h}$, $\mathrm{t} 3 \mathrm{~m}$ ). Estos textos fueron seleccionados porque muestran desempeños representativos de las formas en que los estudiantes enfrentan y tratan de resolver distintas problemáticas con el fin de mejorar la escritura en las recomendaciones. Además, en el Anexo 3, se incluyen la primera y última versiones de las otras tres alumnas (textos: $\mathrm{t} 4 \mathrm{~m}, \mathrm{t} 5 \mathrm{~m}$ y t6m). Para proteger la identidad de los participantes, se sustituyen los nombres mediante el siguiente código de identificación: (t) texto, número asignado a cada estudiante y (h) hombre o (m) mujer.

\subsection{Caso 1: t1m, recomendación de "Nasreddin and the professor"}

\subsubsection{Primera versión}

El primer ejemplo que se analizará es una recomendación sobre la lectura "Nasreddin and the professor" (Hill, 2007: 8-9). En (1) se muestra la primera versión elaborada de este texto. ${ }^{3}$ La alumna que lo elaboró aún no consolida el uso de

3 Al final de cada ejemplo se señala el código de participante como se describió arriba, el número de versión $(1 \mathrm{aV}, 2 \mathrm{aV}, 3 \mathrm{aV}$ y $4 \mathrm{aV})$ y el nombre del texto recomendado con la siguiente clave: 
los pronombres y de los tiempos presente y pasado y maneja un vocabulario suficiente para expresar el propósito, sin embargo, confunde algunos significados y emplea oraciones largas que la llevan a cometer errores sintácticos. Además, manifiesta una intencionalidad en la que trata de persuadir con su nivel de inglés.

(1) My dear young:

If you wont learn english, and amuse oneself this is for you. "Nasreddin and the professor" is a reading very interesting, where you can practice vocabulary and enjoy. In this chapter, a professor and our personage. primary have an appointment, but Nasreddin forget to the professor, and of a way they two say IDIOT. mutually. I'm like more this history and I think so that it's very funny.

¡You can't lost this reading, You have to read! [t1m, 1aV, N\&P]

\subsubsection{Segunda versión}

En (2) se muestra la segunda versión del texto tras la aplicación del corrector ortográfico y gramatical Word, sin el apoyo del profesor:

(2) My dear young:

If you won't learn English, and amuse oneself this is for you. "Nasreddin and the professor" is a reading very interesting, where you can practice vocabulary and enjoy. In this chapter, a professor and our personage-primary have an appointment, but Nasreddin forget to the professor, and of a way they two say IDIOT; Mutually. I'm like more this history and I think so that it's very funny.

¡You can’t lose this reading, you have to read! [t1m, 2aV, N\&P]

Destaca la representación textual de los interlocutores en If you won't y de la alumna, como autora, al expresar su punto de vista, por ejemplo en I think - de este modo, le da fuerza expresiva a su recomendación. Al respecto de la representación textual, me dijo: "yo escribí oneself cuando era yourself porque estaba hablando de 'tú', le hablo al lector". Así intentó representar a los interlocutores, según la perspectiva planteada por Hyland (2005) sobre la conformación de la relación autor y lector. En la fase de indicación de fallas de ortografía o gramá-

N\&P para "Nasreddin and the professor", Personality para "Your personality in the palm of your hand" y N\&MG para "Nasreddin and the Museum Guide".

Estudios de Lingüística Aplicada, año 35, número 65, julio de 2017, pp. 103-137

DOI: 10.22201/enallt.01852647p.2017.65.730 
tica mediante el corrector del programa Word, la alumna resolvió parcialmente dificultades de ortografía y uso de mayúsculas, por ejemplo, en English. Este tipo de correcciones corresponde al estándar textual de aceptabilidad del escrito en ambiente formal y escolar, donde es necesaria la ortografía adecuada. El corrector del programa Word le indicó errores relacionados con puntuación que intentó resolver sin éxito, por ejemplo, al eliminar el punto y sustituirlo por guión en personage-primary, además de la mayúscula y punto y coma en IDIOT; $M u$ tually. Estos aspectos de puntuación impactan en la cohesión textual.

El desempeño de la alumna manifiesta regulación reflexiva e intencionalidad al tratar de resolver las dificultades enfrentadas. Logró resolver cuestiones relacionadas con el estándar de cohesión gramatical, por ejemplo, en la frase You can't lose, pero tuvo dificultades, como en I'm like more this history y en a reading very interesting. El recurso de subrayado de errores del corrector gramatical Word en you won't learn, no le ayudó para reelaborar conforme al sentido que deseaba expresar: you want to learn. Como menciona Beuchot (2015), en ocasiones aquello que aparece escrito no corresponde a lo que el autor trató de expresar. Aquí, lo expresado y detectado con el programa Word está gramaticalmente correcto, sin embargo, no es la intención que la alumna quería expresar. Esta situación evidencia que este recurso del programa es un apoyo relacionado con el estándar de cohesión en el nivel gramatical, pero no con el de coherencia textual. En la asesoría dedicada a revisar la segunda versión, se le comentó a la alumna que esta primera revisión con el corrector del programa Word no sería suficiente para resolver cuestiones de sentido en el vocabulario. Por ejemplo, se le preguntó lo que quería decir en If you won't learn. Entonces, se le explicó que la oración era correcta, pero que no correspondía a lo que deseaba expresar: If you want to learn. En cuanto al estándar de intertextualidad, es decir, relacionar su escrito con el texto recomendado, se le comentó que debía mencionar el autor y el título del libro donde aparece la historia de Nasreddin. Asimismo, se le sugirió que describiera otros detalles del libro: ilustraciones y tipo de lenguaje.

Las reflexiones sobre el desempeño propician, tal como propone Wenden (1986), la toma de conciencia del manejo de recursos textuales. Por ello, se propició la reflexión en la alumna mediante las siguientes preguntas: “¿Qué te pareció esta experiencia de escritura?, ¿por qué?” En su reflexión predominaron los aspectos relacionados con los estándares textuales de cohesión en el aspecto grama- 
tical y de coherencia, al percatarse de usos que requería modificar, no indicados como errores mediante el corrector gramatical Word. Veamos sus comentarios:

Pues me hizo aprender formas diferentes de expresión y mejorar la estructura gramatical porque muchas veces no escribo la persona. Y usualmente no cambio los tiempos verbales ni me fijo en el tiempo en que lo estoy expresando y fui checando error por error y revisándolos con el profesor después de que hice un chequeo con el corrector gramatical de Word y muchas cosas este las omitía.

Posteriormente, se le pidió que escribiera una nueva versión.

\subsubsection{Tercera versión}

En (3) se muestra la tercera versión del texto, modificada después de revisar el subrayado de errores del corrector de Word. Se puede apreciar el interés en tratar de corregir aspectos gramaticales, por ejemplo, en very interesting reading, Nasreddin forget it y I like more this history than others. A pesar de no lograr resolver todas las dificultades y no prestar atención al contenido ni a la información sobre el título del libro, presenta un avance significativo en el desempeño.

(3) My dear young:

If you want to learn English, and amuse yourself, this is for you. "Nasreddin and the professor" is a very interesting reading, where you can practice vocabulary and enjoy. In this chapter, a professor and our primary personage have an appointment, but Nasreddin forget it, and in a certain way they two say IDIOT mutually. I like more this history than others and I think that it's very funny.

¡You can't lose this reading, you have to read it! [t1m, 3aV, N\&P]

Después, otro docente apoyó a la alumna con comentarios escritos y orales acerca de esta tercera versión. Este asesor abordó cuestiones de cohesión gramatical, vocabulario y de los sentidos expresados por las opciones léxicas, mediante una breve explicación y sugerencias. Le propuso a la alumna revisar diccionarios en línea. De igual manera, le recomendó emplear oraciones simples y cortas enlazadas con un conector. 
Posteriormente, se le solicitó a la alumna que tomara en cuenta las experiencias de este ejercicio para escribir la siguiente versión del texto. Se retomó la orientación sobre las opciones de revisión y corrección con el programa Word. Asimismo, se revisaron los aspectos donde la alumna presentó dificultades. Al respecto, se le comentó que debía emplear el tiempo pasado called en la oración The professor was angry and call Nasreddin idiot. Asimismo, que corrigiera el tiempo verbal en have an appointment, but Nasreddin forget it. Se le sugirió presentar la información en pasado con los verbos had y forgot. Se le insistió sobre el título del libro y el nombre del autor.

\subsubsection{Cuarta versión}

En (4) se muestra la cuarta y última versión del texto, tras recibir retroalimentación del asesor y la de otro docente de la mediateca. La alumna empleó oraciones cortas, así evitó errores de cohesión, y cambió palabras y frases por otras expresiones más sencillas, por ejemplo, al sustituir our primary personage por, simplemente, Nasreddin. Resolvió cuestiones ortográficas y gramaticales como resultado de la revisión y la reflexión sobre los comentarios y las sugerencias. Además, incorporó elementos de intertextualidad al relacionar el escrito con el texto recomendado. Al informar sobre el autor y el título, la alumna escribió: "In the book 'Nasreddin Ten Stories' of the author Jennifer Gascoigne, illustrated by Tulli Masinari, you will find a easy reading with pictures very funny. You will want to read it immediately".

(4) ¡Nasreddin and the Professor!

Dear students:

If you want to learn English, and amuse yourself this is for you. In the book "Nasreddin Ten Stories" by Jennifer Gascoigne, illustrated by Tulli Masinari, you will find an easy reading with very funny pictures. You will want to read it immediately. "Nasreddin and the professor" is a very interesting story, because you can practice vocabulary and enjoy the moral. In this chapter, a professor and Nasreddin have an appointment, but Nasreddin forgets it. The professor was angry and called Nasreddin idiot. But with Nasreddin's intelligence the real idiot was the professor.

I like this story and I think that it is very funny.

You can't lose this reading, you have to read it! [t1m, 4aV, N\&P] 
Como comentarios a esta última versión, se le hicieron las observaciones correspondientes de ortografía, indicadas por el corrector ortográfico y se le hizo notar el error en el orden de la frase pictures very funny. La alumna atendió estas observaciones sin dificultad y mostró avance en su desempeño; sólo le faltó corregir detalles: emplear miss en lugar de lose; tomar en cuenta la sugerencia de escribir los tiempos verbales en pasado en los casos de have, forgets, y precisar a qué tipo de enseñanza hizo referencia en la expresión enjoy the moral. A pesar de esto, la última versión cumple los estándares de aceptabilidad y situacionalidad, puesto que responde a las expectativas de un entorno escolar. Oxford (1990) considera este tipo de desempeño como el resultado de estrategias compensatorias para resolver dificultades de manera reflexiva y autorregulada. La alumna comentó que en su plan de asesorías le interesaba trabajar la gramática, el vocabulario y la ortografía. En su reflexión predomina la consideración de aspectos relacionados con los estándares de cohesión y coherencia.

\subsection{Caso 2: $t 2 h$, recomendación de "Your personality in the palm of your hand?"}

\subsubsection{Primera versión}

El alumno seleccionó un texto de un nivel intermedio, con un tema y lenguaje de mayor complejidad: "Your personality in the palm of your hand?" (Purpura \& Pinkley, 1992: 51-52). El estudiante usa un vocabulario que le permite comprender la lectura, sin embargo, tiene dificultades gramaticales en la producción oral y escrita. En (5) se observa la primera versión:

(5) I don't belive in the reading of hands, because it donest are exactly. I thing this is for idiots who got no trust in themselves and want to know they future because they thing the future is dark for them and they search a way out of that fate.

I think, the people who trust in it need to belive in themselves to got a TRUE change on they lives, making good things in they lives and begin to be people whit firm proposals and goals and droop that stupid thought for got a REAL change in they lives. [t2h, $1 \mathrm{aV}$, Personality] 


\subsubsection{Segunda versión}

Antes de revisar la primera versión del texto con el programa Word y de recibir sugerencias, el alumno comentó que retomaría su recomendación, sin embargo, no realizó cambios a lo ya escrito, solo agregó información, tal como podemos observar en la segunda versión que se presenta en (6). Esta actitud podría interpretarse como un indicador de seguridad por parte del estudiante.

(6) I don't belive in the reading of hands, because it donest are exactly. I thing this is for idiots who got no trust in themselves and want to know they future because they thing the future is dark for them and they search a way out of that fate.

I think, the people who trust in it need to belive in themselves to got a TRUE change on they lives, making good things in they lives and begin to be people whit firm proposals and goals and droop that stupid thought for got a REAL change in they lives.

If you belive in that, CHANGE YOUR MIND, you're wrong, don't search easy ways to exit your problems like this, make accions and go to face your problems to solve it, not to run away of they. [t2h, $2 \mathrm{aV}$, Personality]

Después se le dio orientación al alumno en la fase de revisión y sugerencias aportadas por el corrector ortográfico y gramatical del programa Word bajo la opción de subrayado de errores. El alumno identificó errores ortográficos, por ejemplo, en los términos believe y think. Se le pidió que tomara en cuenta todas las actividades realizadas para escribir la siguiente versión.

La asesora de lengua que apoyó esta actividad hizo sus observaciones oralmente. Se le pidió al alumno que prestara atención y tomara notas. Las observaciones se centraron en la cohesión textual gramatical, principalmente en el uso de verbos y pronombres. Ella enfatizó la adecuación del lenguaje al de un entorno académico formal para no usar expresiones fuertes que pudieran hacer sentir mal al lector, como: I think this is for idiots, droop that stupid thought y you're wrong. Esta observación responde a los estándares de aceptabilidad del lenguaje y al de situacionalidad de acuerdo con la perspectiva de De Beugrande y Dressler (1997). Esta toma de conciencia es fundamental, ya que es común que los estudiantes escriban en registros que consideran apropiados en todos los contextos. Asimismo, enfatizó que el uso de expresiones como idiots y stupid no es adecuado en un entorno académico formal, ya que pueden resultar ofensivos para el lector. 
Una vez que el estudiante recibió la retroalimentación de la asesora, se le apoyó con otras observaciones y sugerencias, retomando cuestiones de gramática y vocabulario. Se le comentó que su manera de opinar en primera persona del singular le daba fuerza expresiva para presentar sus puntos de vista. Además, se le recomendó que considerara a los interlocutores: "Toma en cuenta a quien estás escribiendo y dirígete de manera directa a tus lectores, hazles la recomendación a ellos, tómalos en cuenta porque en tu escrito estos no aparecen". Hyland (2005) considera esta manera de tener presente al lector mediante las representaciones textuales del autor y de los interlocutores como prácticas reflexivas fundamentales. Así, los autores asumen una "voz textual", en este caso, para convencer a otros sobre la lectura recomendada.

El alumno se mostró sorprendido por las observaciones recibidas, y destacó su mejor desempeño oral. Se le propuso que escribiera sus impresiones, y decidió hacerlo en inglés, tal como vemos a continuación:

My reflections about my perfomance.

What I learned? Wow, I don't know where begin. That comment was hard to assimilate for me, because I feel at the first like a man whit a strong knowledge, but after her comment, I feel too awkward because my personal writing and grammar are bad than I expected.

I learned I need be careful whit a lot of things on grammar and improve my writing because it not coincides to my skills on speaking and talking. I want to learn more about the English and whit that got enough for go to New Zeland or Australia.

Today I learnd to write for another person, not only giving my opinion, because when $\mathrm{i}$ write that kind of text, i hope there can be some change in the minds of others, not only give my point. [t2h]

Estas reflexiones relacionadas con el desempeño propio dan cuenta del proceso de construcción de identidad, propuesta por Ivanič (1998), sobre lo que representa para los estudiantes la valoración del desempeño al recibir retroalimentación en una situación comunicativa escolar. 


\subsubsection{Tercera versión}

En (7) se muestra la tercera versión corregida después de emplear el corrector de Word. El alumno centró su atención en aspectos de cohesión en el nivel gramatical. La retroalimentación le ayudó a examinar su propia intencionalidad relacionada con el estándar de coherencia al emplear la referencia pronominal. Intentó resolver aspectos gramaticales, pero tuvo dificultades en la corrección de fragmentos como: it doesn't exactly, them future, you need change y you got a wrong. De igual forma, no se dio cuenta del problema de coherencia en la expresión face your problems to solve it. Esta sería gramaticalmente correcta en otro contexto, pero en este el pronombre it no corresponde al plural requerido para la expresión "your problems". El estudiante aclaró lo que deseaba decir. Cuando se le explicó el uso de los pronombres it y them realizó la corrección. De este modo se percató, en este caso, de la necesidad de prestar atención a aspectos de cohesión gramatical y coherencia en el manejo de contenidos que no podía resolver con el apoyo del corrector gramatical de Word. Se le comentó que tenía que apoyarse en sus conocimientos y también en otros recursos de consulta gramatical disponibles en el acervo.

\section{(7) CORRECTION:}

I don't believe in the Reading of Hands because it doesn't exactly. I think this is for people who don't trust in them and want to know them future and them think the future is dark for them and search a way out of that fate.

If you believe in the reading of hands I think you need change a little your mind, you got a wrong, don't look for easy ways to exit from your problems like these, do actions and face your problems to solve it, don't run away of them. [t2h, $3 \mathrm{aV}$, Personality]

Cuando se detectó la dificultad que implicaba para el alumno resolver la oscilación pronominal entre they y them, se le recordó y explicó los usos de este pronombre como sujeto y como posesivo, así como la necesidad del infinitivo en la construcción you need change. Por el momento, no se le hizo la observación sobre el orden de las palabras en la oración change a little your mind, ni sobre la puntuación necesaria para introducir la oración subordinada condicional. Se le comentaron aspectos relacionados con los estándares de informatividad e inter- 
textualidad. En este sentido, se le dijo que era necesario abordar el contenido, el título del libro y el autor.

Un ejemplo de la manera en que se apoya a los estudiantes en la autorregulación del desempeño es el acompañamiento que se le dio en los intentos para corregir la expresión it donest are exactly. La reflexión sobre las modificaciones realizadas favoreció el avance en la intencionalidad. En este caso, el estudiante tenía que resolver el uso del verbo correcto y del adjetivo exact. Cuando eliminó el verbo are, mantuvo el problema en it doesn't exactly. Se le comentó esta situación y se le explicó el uso del verbo to be en singular; paralelamente, se le recordó el uso de doesn't y se le mostró la distinción entre adverbio y adjetivo, correspondiente a exact y exactly. Se le propuso que empleara un adjetivo o una frase para explicar su idea. Sin embargo, en ese momento no logró resolver este problema.

\subsubsection{Cuarta versión}

En la cuarta versión, que se observa en (8), el alumno incluyó un título para su recomendación: "My opinion of Reading of the Hands". En la intencionalidad de la recomendación predominó su punto de vista sobre el tema, pero no proporcionó información suficiente sobre el contenido de la lectura, no mencionó al autor y solo hizo referencia de manera ambigua a la lectura recomendada en: go and read that text. En este sentido, su texto no cumple con los estándares de informatividad e intertextualidad, de modo que el lector no tiene los elementos suficientes para la comprensión. Se le comentó esto al alumno, pero en ese momento no quiso escribir otra versión. Entonces, se le sugirió trabajar esas cuestiones en asesorías posteriores.

(8) My opinion of Reading of the Hands:

I don't believe in the Reading of the Hands because it isn't exactly. I think this is for people who don't trust in them and want to know their future and they think the future is dark for them and they search a way out of that fate.

If you believe in the reading of hands I think you need to change a little your mind. You've got a wrong idea. Don't look for easy ways to avoid or exit from your problems like these, do actions and face your problems to solve them, don't run away of them.

Anyways, this is my opinion, maybe you've got another idea, go and read that text, in fact is interesting and good to improve your reading. [t2h, 4aV, Personality] 
El alumno consideró la estructura textual al incorporar un cierre para motivar a la lectura, en el que omite el sujeto: go and read that text, in fact is interesting and good to improve your reading. Se le comentó sobre la necesidad de hacer ejercicios para practicar el uso de pronombres. El alumno prestó atención a las selecciones léxicas al eliminar un lenguaje fuerte o agresivo. En su texto predomina la consideración de recursos correspondientes a los estándares de cohesión, coherencia, situacionalidad y aceptabilidad.

A pesar de que la última versión no cumple con los criterios de informatividad e intertextualidad, es evidente la intencionalidad en la revisión, edición y reflexión sobre la escritura a partir de la regulación del desempeño. El alumno valoró su manejo de recursos textuales, y en su intencionalidad empleó estrategias alternativas o compensatorias, tal como concibe Oxford (1990). Esta situación pone de manifiesto las reflexiones y concepciones sobre el desempeño, a partir de experiencias de regulación de la escritura y de retroalimentación durante la revisión y la edición textuales. El estudiante estuvo de acuerdo en trabajar aspectos gramaticales en asesorías posteriores, puesto que aún quedaron cuestiones por resolver, por ejemplo, la sugerencia con respecto al uso de adjetivo en la oración it isn't exactly, la ausencia de sujeto en in fact is interesting y la selección léxica en ways to avoid or exit from your problems. El alumno enfatizó que en las asesorías trabajaría solamente sus habilidades de escritura, ya que, comentó, en la expresión oral y en la comprensión lectora tenía un mejor desempeño. Esta insistencia en destacar su mejor desempeño oral y en la lectura manifiesta una actitud relacionada con la construcción de identidad, tal como propone Ivanič (1998), sobre lo que impacta y representa para el estudiante tomar conciencia de su desempeño y descubrir que puede mejorar determinadas cuestiones a partir la experiencia de revisión y edición.

\subsection{Caso 3: $t 3 m$, recomendación de "Nasreddin and the museum guide"}

\subsubsection{Primera versión}

En este ejemplo, se revisa la recomendación de la lectura "Nasreddin and the museum guide" (Hill, 2007: 16-17). La alumna tiene conocimiento del presente y pasado simples. Manifiesta un proceso de aprendizaje gradual de los recursos 
de escritura en las versiones redactadas. En (9) se presenta la primera versión de este texto.

(9) Nasreddin Ten stories

Retold by Jennifer Gascoigne

Ilustrate by Jullid Masinar

Storie No. 8

"Nasreddin and the museum guide"

Nasreddin is the old man and visit the museum, and in the visit, take the paper of the guide, the guide angry, because Nasreddin funing.

The book present

* Beautiful illustrations

* Good stories

The book is very interesting. [t3m, $1 \mathrm{aV}, \mathrm{N} \& \mathrm{MG}]$

En la retroalimentación, la alumna prestó atención a las indicaciones mostradas mediante la aplicación del corrector de Word, centradas en cuestiones ortográficas, como en el caso de la palabra storie, y gramaticales, como en Nasreddin funing e ilustrate by. La alumna comentó que necesitaba corregir estos aspectos, sin embargo, no se percató de otras dificultades, no indicadas por el corrector ortográfico y gramatical de Word. En este caso, la herramienta no señaló indicaciones sobre los verbos, debido a que eran correctos en la forma imperativa en que aparecían, como en las oraciones: visit the museum y take the paper of the guide. Sin embargo, la intención de la alumna no corresponde a la del imperativo revisado por el corrector del procesador de textos. Esto es una evidencia más de que esta herramienta es de utilidad, siempre y cuando se realice también la reflexión y regulación del conocimiento y desempeño gramaticales.

En este caso, se le sugirió a la alumna emplear el pasado en las siguientes oraciones: Nasreddin is the old man, take the paper of the guide, visit the museum y se le señaló la necesidad de usar un verbo en la frase the guide angry. Se le propuso que escribiera oraciones cortas con la estructura sujeto + verbo + complemento, por ejemplo: "Nasreddin was an old man. He visited a museum". Así podría evitar dificultades relacionadas con el estándar textual de cohesión.

Se le recomendó a la alumna que expresara su opinión del texto y que tomara en cuenta a los lectores, dirigiéndose a estos para invitarlos a leer el relato. De 
esta forma, lograría la representación textual del autor y de los interlocutores. La alumna realizó modificaciones parciales a partir de las observaciones anteriores.

\subsubsection{Segunda versión}

En (10) se presenta la segunda versión redactada por la alumna. En su escrito podemos apreciar el avance gradual de la intencionalidad y del desempeño en la organización de la información. La alumna intentó regular la cohesión textual y evitó complicaciones gramaticales al emplear oraciones más cortas, como Nasreddin is the old man, He visit the museum. Presentó avance en los tiempos verbales en was angry, assumed y had a funny attitude, a pesar de que no escribió correctamente la forma del pasado del verbo visit y el participio en Ilustrate. Esta situación evidencia el proceso gradual de aprendizaje de los tiempos verbales en su desempeño de nivel inicial.

\section{(10) NASREDDIN}

Ten stories

Storie No. 8

"Nasreddin and the museum guide"

Retold by Jennifer Gascoigne

Ilustrate by Jullid Masinar

Nasreddin is the old man. He visit the museum, and in the visit, assumed the role of the guide, the guide was angry, because Nasreddin had a funny attitude.

I think that the book is interesting and funny.

¡YOU! Will hav the very good experiens, because read ten stories very funning

The book present

* Beautiful illustrations

* Good stories

The book is very interesting. [t $3 \mathrm{~m}, 2 \mathrm{aV}, \mathrm{N} \& \mathrm{MG}]$

Se puede observar aquí la representación textual del autor y los interlocutores, cuando la alumna opina usando la primera persona del singular en I think y cuando se dirige a los lectores sobre la experiencia de lectura en ;YOU! Will hav the very good experiens. Se le sugirió que empleara minúscula en el auxiliar modal Will, sin embargo, me explicó que trató de atraer a los lectores mediante 
el uso del pronombre en mayúsculas y con signos de admiración. Apreciamos un esfuerzo por mejorar el léxico en el uso de funning y la expresión corregida funny. Este intento por cuidar la ortografía y el léxico constituye una regulación de recursos relacionados con los estándares de adecuación y situacionalidad del escrito en un contexto escolar.

Se le recomendó a la alumna evitar la repetición en las frases consecutivas the role of the guide, the guide y que proporcionara más información sobre la lectura, sin contar aspectos de más. Asimismo, se le sugirió que empleara una oración simple, sin omitir el sujeto y el verbo en construcciones como because read ten stories very funning. Además, se le hicieron observaciones sobre el uso del tiempo presente en The book present y sobre la ortografía de las expresiones hav y experiens, marcadas por el corrector del procesador Word. La estudiante resolvió gradualmente estos aspectos en la revisión y edición del texto.

\subsubsection{Tercera versión}

En la tercera versión que podemos observar en (11), la alumna avanzó en el manejo del pasado y en la elección del léxico. Resolvió las complicaciones de estructuración gramatical con el empleo de oraciones simples enlazadas con comas. En cuanto al estándar de informatividad, incorporó contenidos para interesar al lector cuando explicó: He visited the museum four years before.

Al modificar el léxico, la estudiante se enfrentó al problema del uso del artículo indefinido en la frase a old man y a ciertas cuestiones ortográficas en Ilustrated e inteligent. También se percató de esta situación por la indicación del corrector ortográfico y gramatical, sin embargo, no corrigió la preposición en whit this story. La palabra whit funciona como sustantivo ('pizca', 'ápice', 'poquito'), por ello el corrector del procesador Word no la marcó como error. Se le pidió que corrigiera la preposición y se le enfatizó acerca de los alcances y limitaciones del corrector ortográfico y gramatical de Word. Finalmente, la alumna hizo las modificaciones requeridas con los criterios de selección de léxico y de coherencia textual.

\section{(11) NASREDDIN}

Ten stories

Story No. 8 
"Nasreddin and the museum guide"

$$
\begin{aligned}
& \text { Retold by Jennifer Gascoigne } \\
& \text { Ilustrated by Jullid Masinar }
\end{aligned}
$$

Nasreddin was a old inteligent man. He visited the museum, and in the visit, he assumed the role of the guide, he was angry, because Nasreddin had a funny attitude. He visited the museum four years before.

I think that the book is interesting and funny.

¡YOU! Will have a very good experience whit this story

The book presents

* Beautiful illustrations

* Good stories

The book is very interesting. [t $3 \mathrm{~m}, 3 \mathrm{aV}, \mathrm{N} \& \mathrm{MG}]$

\subsubsection{Cuarta versión}

En la cuarta versión que se presenta en (12), se puede observar el avance de la alumna en la intencionalidad y autorregulación del desempeño, puesto que resolvió aspectos ortográficos y gramaticales. Su texto cumple con los estándares de adecuación y situacionalidad y proporciona elementos de informatividad e intertextualidad, además es perceptible la representación textual del autor y de los interlocutores.

\section{(12) NASREDDIN}

Ten stories

Story No. 8

"Nasreddin and the museum guide"

\section{Retold by Jennifer Gascoigne \\ Illustrated by Jullid Masinar}

Nasreddin was an old intelligent man. He visited the museum, and in the visit, he assumed the role of the guide, he was angry, because Nasreddin had a funny attitude. He visited the museum four years before.

I think that the book is interesting and funny.

¡YOU! Will have a very good experience with this story. 
The book presents

* Beautiful illustrations

* Good stories

The book is very interesting. [t $3 \mathrm{~m}, 4 \mathrm{aV}, \mathrm{N} \& \mathrm{MG}]$

Se le comentó a la alumna acerca de la necesidad de eliminar la coma en he was angry, because Nasreddin had a funny attitude, debido a que producía confusión. La corrección le dio fluidez a la idea, es decir, mejoró la cohesión y la coherencia.

La alumna comentó por escrito que las revisiones y correcciones con el corrector de Word y las sugerencias fueron útiles para tomar en cuenta su desempeño y lo que podía mejorar.

Opino que es bueno tener dos puntos de vista diferentes para así darme cuenta en que puedo mejorar mi manera de expresar mi recomendación, en general fue buena la experiencia pues es bueno saber que mi ingles no es del todo mal y que con esfuerzo lo puedo perfeccionar. Con Word pude notar que hacia correcciones inadecuadas (ten stories), pero que también algunas que en relación con el profesor eran iguales, al igual pude notar que en las dos correcciones tenía problemas con los verbos en pasado y también repasar los verbos en presente. $[\mathrm{t} 3 \mathrm{~m}]$

Este tipo de opiniones sobre el desempeño, la retroalimentación y las prácticas de revisión y edición textuales favorecen la toma de conciencia sobre el manejo de recursos de textualización y la búsqueda de estrategias para resolver dificultades en cada una de las versiones redactadas.

\section{ConClusión}

El seguimiento de la redacción de las recomendaciones de textos de la mediateca permitió que los estudiantes autorregularan sus desempeños por medio de la revisión y edición de las versiones escritas. El ejercicio muestra que los estudiantes requieren prácticas que propicien la valoración autocrítica del desempeño, de manera tal que, al recibir retroalimentación, manifiesten intencionalidad en la redacción en inglés. Los escritos presentan evidencias de la reflexión y autorregulación necesarias para resolver aspectos relacionados con los estándares 
de textualidad, así como del reconocimiento y el manejo del tipo de texto requerido y la representación textual del autor y los interlocutores.

En los textos predomina la atención prestada a la ortografía y la gramática. Esto se debe, en gran medida, al trabajo realizado con el corrector ortográfico y gramatical del procesador de textos Word. La principal dificultad para los alumnos fue tratar de resolver cuestiones formales y gramaticales relacionadas con la coherencia y la cohesión. Los alumnos experimentaron la reflexión crítica y de autorregulación en sus intentos por resolver estructuras gramaticalmente correctas, conforme al corrector ortográfico y gramatical del programa Word, pero que no correspondían a lo que deseaban expresar. Así, apreciamos la intencionalidad y la regulación del desempeño en busca de dar cohesión y coherencia al texto. En este punto, la asesoría fomentó la reflexión sobre la lengua y las decisiones sobre los criterios y recursos textuales para mejorar la escritura. De este modo, los estudiantes tomaron conciencia de aspectos que pueden trabajar en asesorías posteriores.

Los estudiantes enfrentaron dificultades relacionadas con los estándares de informatividad e intertextualidad al momento de proporcionar los contenidos necesarios y vincular el texto redactado con el recomendado. Al tomar en cuenta a los interlocutores, consideraron la adecuación y la situacionalidad del vocabulario, así como las expresiones pertinentes en un contexto formal y escolar, es decir, la recepción del texto por los lectores en determinado contexto.

El diseño metodológico en la sesión grupal inicial, las consideraciones sobre el tipo de texto para recomendar una lectura y los acuerdos ayudaron a la reflexión sobre el desempeño. En los acuerdos, los estudiantes manejaron conocimientos sobre las características de la recomendación escrita de una lectura. De esta forma, cuando los alumnos revisan escritos de quienes anteriormente han recomendado lecturas, conforman sus propios modelos textuales a partir de valorar distintos desempeños. Además, los estudiantes se percataron del impacto de las retroalimentaciones de revisión mediante el corrector del programa Word, las sugerencias y observaciones del asesor y, cuando fue posible, de otro docente. Así, los estudiantes ampliaron sus recursos y acervos de prácticas de textualidad.

En las opiniones sobre las actividades de revisión y edición, los estudiantes manifestaron algunas concepciones y valoraciones sobre sus habilidades que no correspondían a su desempeño real. Las actividades les ayudaron a identificar y tomar conciencia sobre los aspectos que requerían mejorar. De este modo, emplearon estrategias alternativas o compensatorias para resolver distintas dificul- 
tades. Los estudiantes manifestaron actitudes y elementos de identidad sobre lo que implica la práctica de escritura y las reacciones ante la retroalimentación. La labor del asesor es fomentar una actitud favorable para que los estudiantes se animen a escribir, a recibir sugerencias sin sentirse criticados o señalados y a editar sus textos con la idea de mejorarlos. La toma de acuerdos y la interacción en la asesoría para recibir retroalimentación favorecen la socialización de las experiencias de autorregulación en el manejo de conocimientos, recursos y decisiones de textualización, así como la práctica de estos conocimientos textuales. Al tomar conciencia del desempeño, los estudiantes pueden evaluarlo y autorregularlo.

En esta ocasión, se aplicó la propuesta para la escritura en inglés en asesorías individuales y en pequeños grupos en la mediateca, sin embargo, la metodología puede aplicarse en otros contextos en lengua materna y en otras lenguas extranjeras. Para ello, es necesario adaptar el diseño metodológico en cada situación escolar, por ejemplo, en grupos de trabajo en el aula y horas de estudio, así como asesorías presenciales y a distancia. El enfoque de autorregulación del desempeño mediante la revisión y edición reflexiva y retroalimentada se justifica debido a las dificultades de los estudiantes para la escritura académica en las lenguas materna y extranjera. En futuras investigaciones esta propuesta se puede implementar en la caracterización de los desempeños lingüísticos en las perspectivas de la intencionalidad y la autorregulación en las prácticas de revisión y edición de textos.

\section{REFERENCIAS}

Bausela Herreras, Esperanza (2007). Relación entre autorregulación y autoconocimiento de la escritura con la coherencia y productividad de un texto. Cuestiones pedagógicas, 18, 257-270.

Beuchot, Mauricio (2015). Elementos esenciales de una hermenéutica analógica. Diánoia, $60(74), 127-145$.

Castelló, Montserrat; Bañales Faz, Gerardo, \& Vega lópez, Norma Alicia (2010). Enfoques en la investigación de la regulación de escritura académica: estado de la cuestión. Electronic Journal of Research in Educational Psychology, 8(3), 1253-1282. Recuperado de http://www.redalyc.org/pdf/2931/293122000015.pdf

Council of Europe for Cultural Cooperation Education Committee (2001). Common European Framework for Languages: Learning, teaching, assessment. Estrasburgo: Cambridge University Press.

Estudios de Lingüística Aplicada, año 35, número 65, julio de 2017, pp. 103-137

DOI: $10.22201 /$ enallt.01852647p.2017.65.730 
Crook, Marie, \& Dixey, Kay (Eds.) (2000). The princess and the frog. Harlow: Pearson Education.

De Beugrande, Robert Alain, \& Dressler, Wolfgang Ulrich (1997). Introducción a la lingüística del texto. Barcelona: Ariel.

Dickinson, Leslie (1987). Self-instruction in language learning. Cambridge: Cambridge University Press.

Escuela Nacional Preparatoria (1996). Programa de inglés IV lengua extranjera. México: Universidad Nacional Autónoma de México.

Hill, Robert (Ed.) (2007). Nasreddin ten stories. Génova: Black Cat.

Hobart, Ruth (Ed.) (2006). Cinderella. Génova: Black Cat.

Hyland, Ken (2005). Metadiscourse. Londres: Continuum.

IVANIČ, Roz (1998). Writing and identity: The discoursal construction of identity in academic writing. Filadelfia: John Benjamins.

Martín Leralta, Susana (2006). La integración de la competencia estratégica en el currículo de lengua extranjera. Estudios de Lingüística Universidad de Alicante, 20, 233-257.

OXFORD, REBECCA L. (1990). Language learning strategies: What every teacher should know. Boston: Heinle \& Heinle/Cengage.

PAlAPANidi, Kiriakí (2016). La enseñanza de las estrategias compensatorias. Revista Nebrija de Lingüística Aplicada a la Enseñanza de las Lenguas, 10(2). Recuperado de http://www.nebrija.com/revista-linguistica/la-ense\%C3\%B1 anza-de-las-estrategias-compensatorias

Purpura, James E., \& Pinkley, Diane (1992). On Target 2. Illinois: Scott Foresman.

ToRre Puente, JuAn CARlos (1992). Aprender a pensar y pensar para aprender. Madrid: Narcea.

Wenden, Anita (1986). Helping language learners think about learning. English Language Teaching Journal, 40(1), 161-175.

Estudios de Lingüística Aplicada, año 35, número 65, julio de 2017, pp. 103-137

DOI: $10.22201 /$ enallt.01852647p.2017.65.730 


\section{ANEXos}

Anexo 1. Lecturas seleccionadas por los alumnos

"Nasreddin and the professor" (Hill 2007: 8-9), lectura de nivel básico seleccionada por la alumna $11 \mathrm{~m}$ :

A professor made an appointment with Nasreddin to talk about a difficult problem. "I'll come to your house at 12 o'clock tomorrow," he said. When he arrived at Nasreddin's house, he knocked again but there was still no reply. $\mathrm{He}$ looked through the windows but he couldn't see anybody. The professor was angry. He took a piece of chalk and wrote the word IDIOT on the door in big letters. Nasreddin returned home later and he saw it. "Oh dear!" he said. "My appointment with the professor!" He immediately ran to the professor's house. "I'm very sorry," he said. "I completely forgot it. I only remembered it when I saw your name on my door!"

"Your personality in the palm of your hand?" (Purpura \& Pinkley, 1992: 50-51), lectura de nivel intermedio seleccionada por el alumno t $2 \mathrm{~h}$ :

Throughout history, people have been fascinated by the mysteries of the human personality. In their efforts to find out how and why humans differ from each other, people have looked for answers in the stars, in the analysis of handwriting and drawings, in the study of the shape of the head, and in the lines and shapes of the hand.

Anyone can look at a human hand and deduce some facts about the owner. For example, a hand with blisters and calluses tells us its owner does hard physical labor, while a soft hand with long nails tells us the owner does not. But some people have gone way beyond that simple step to a much more exotic way of analyzing a person's character. Through the ages, these analysts have identified and studied different lines and shapes (called mounts) in the palm of the hand and have connected them to certain human personality traits.

Experts in palm reading identify nine separate lines in the human palm. The length and clarity of these lines determine certain aspects of personality. For instance, three of the most important lines are the life line, the head line, and the heart line. A long life line shows that the owner will keep on living a health life to a very old age. The head line is related to intelligence; a long, 
curved line shows that the owner is used to thinking imaginatively. The heart line shows love and affection. A short line indicates that the owner has problems expressing affection; in contrast, a long strong line shows that the owner enjoys having passionate relationships.

In addition to identifying lines, readers also look at nine mounts, rounded parts of the palm, that indicate other character traits. These mounts can be flat, round, or very developed. For example, three of the important mounts are the mount of Venus, the mount of the moon, and the mount of Upper Mars. A flat mount of Venus indicates poor health. If the mount of Venus is round, it shows that the owner works hard at having a healthy mind and body through exercise and correct eating. It also indicates a love of being with and helping other people. The owner of a round mount of the moon loves traveling and has a sensitive nature. A strongly developed mount of the moon can indicate creative thinking. A person with a flat mount of Upper Mars can't help believing what other people say; the owner trust people and easily does what others suggest. A very developed mount of Upper Mars, however, indicates that the owner has difficulty in controlling angry feelings and other strong emotions.

After interpreting all the lines and mounts, experts in palm reading say they can then describe a person's personality. (Of course, people who have flat mount of Upper Mars will believe what the palm reader says, people who have round ones probably won't!)

"Nasreddin and the museum guide" (Hill 2007: 16-17), lectura de nivel básico seleccionada por las alumnas $\mathrm{t} 3 \mathrm{~m}$ y $\mathrm{t} 4 \mathrm{~m}$ :

One day Nasreddin was in a museum with some tourists. The museum guide showed them a pot and said. "This pot is five thousand years old." "Excuse me," said Nasreddin politely, "but I think it's five thousand and four years old." The tourists were surprised but the guide was a little angry. He took them to another room and showed some gold coins.

"These coins are three thousand years old," he told them. "Three thousand and four years old," said Nasreddin.

Now the guide was very angry. "How can you know the exact date?" he asked. Nasreddin smiled and replied. "It's very easy. You said the coins were three thousand years old when I was here four years ago!" 
"Nasreddin and the rock" (Hill 2007: 20-21), lectura de nivel básico seleccionada por la alumna t5m:

Nasreddin had a lot of friends. Some of them were old and some of them were young. One day he said to them, "Old men are more intelligent than young men."

"That's true," his old friends replied. "But young men are stronger than old men," said one of his young friends.

"I don't agree," said Nasreddin. "I am still as strong as when I was a young."

"How is that possible?" his friends asked. "Explain that, please, Nasreddin!"

"Well," he said. "Can you see that big rock over there?"

They all looked at the rock.

"When I was a young man, I tried to move it but I couldn't," explained Nasreddin. "I am an old man now and when I try to move it, I still can't."

Nasreddin ten stories (Hill, 2007), libro completo, seleccionado por la alumna t6m. ${ }^{1}$

Anexo 2. Ejemplos de recomendaciones escritas por estudiantes en la mediateca en el ciclo escolar anterior

Recomendación de alumna sobre "Cinderella" (Hobart, 2006):

Do you like love stories where dreams come true? So Cinderella is the perfect book for you!

A full history of magic that you will surely love. It's about a girl who is mistreated by her stepmother and her two stepsisters, but in a dance she will meet the love of her life; but it will only be for that night... so discover what happens with Cinderella. You can find this book in mediateca and you will really enjoy it!

1 Disponible en http://www.blackcat-cideb.com/english-catalogue/163-nasreddin-tenstories-9788853006998.html

Estudios de Lingüística Aplicada, año 35, número 65, julio de 2017, pp. 103-137

DOI: 10.22201/enallt.01852647p.2017.65.730 
Recomendación de alumna sobre "The princess and the frog” (Crook \& Dixey, 2000):

Commentary

Ladies and gentlemen, my name is tm8 and now I am going to speak you about the book "The princess and the frog". This book talks about the fulfilled promises we make. Help to fulfill people and animals realize a dream. In this case the frog was a prince who was bewitched by a witch, who told him that if he spent three days sleeping on the pillow of a princess and eat on her plate the spell would break in that moment the frog will become the prince again. The princess felt disgusting with the frog, but in those 3 days, the princess came to appreciate it. Suddenly, the third day she saw the frog didn't see the frog on her pillow. But she hears a voice in front of her and a handsome young prince. $i$ it was the frog become prince!! He asked her who was now a prince to marry her and they lived happily.

I recommend more this book because we can found a images, values, many lessons and more. No matter the appearance of others, if they need help. We promise to help them, we must fulfill the promise and both will be happy.

¡Do not waste time and come to read!

Autor: Mrs. Marie Crook. Series Editors: Annie Hughes and Melanie Williams.

Thank you

Atte: $\mathrm{t} 8 \mathrm{~m}$

Anexo 3. Primera y última versión de las otras tres alumnas

Alumna $4 \mathrm{~m}$

Versión inicial

Nasreddin and the museum guide

How would you feel if someone is interrupt you every 5 minutes?

You would be happy? This happen to the museum guide with Nasreddin, Nasreddin is a little annoying in this short story, but he is very funny. You have to read this story in the ten stories of Nasreddin retold by Jennifer Gascoigne, you will have a big smile at the end of this little book. 
Versión final

How would you feel if someone interrupts you every 5 minutes?

Would you be happy? This happens to the museum guide with Nasreddin. $\mathrm{He}$ is a little annoying in this short story, but he is very funny because he is very sarcastic with the information. You have to read this story in ten stories of Nasreddin retold by Jennifer Gascoigne. You will have a big smile at the end of this little book.

Alumna $\mathrm{t} 5 \mathrm{~m}$

Versión inicial

Hello!

I read Nasarddin ant the Rock is at the History fabulous

Is ironic, the friends hhe Nasarddin repeat at Nasarddin not is strong, he is old and don't can move a the rock and don't can the young explein nasarddin.

Última versión [No se presentó a realizar los cambios para lograr una versión final]

You have read Nasardin. The history es exelent. the nasarddin's friends are incredulous. his friends say nasarddin is not strong. Nasarddin demostreid at falso argument. He ejemplific with a big rock. He can't move at the rock in the moment. He can't move the rock in the youth.

Alumna t6m

Versión inicial

Nasreddin Ten Stories

If you enjoy to read, or learn to the others experiences, or if you just want to have a enterteinment time, the stories of Nasreddin are perfect to you. In his book there are 10 fantastic short stories, just with one page of this incredible book you can learn to the most wise men ever. You can use the moral messages that Nasreddins stories give you in your life, or maybe you can give a lesson to someone to need it.

Estudios de Lingüística Aplicada, año 35, número 65, julio de 2017, pp. 103-137

DOI: 10.22201/enallt.01852647p.2017.65.730 
Versión final

If you enjoy reading, or learning from other's experiences, or if you just want to have a good time, the stories of Nasreddin are perfect for you. In his book there are 10 fantastic one-page short stories of this incredible book. You can learn from the wisest man. You can use the moral messages in Nasreddin's stories. They can help you in your life, or maybe you can give a lesson to someone that needs it. 
